\title{
SIMULATION OF UPPER LIMB MOVEMENTS
}

\author{
Ing. Filip UHERČÍK, doc. Ing. Branislav HUČKO, PhD. \\ Institute of Applied Mechanics and Mechatronics, Faculty of Mechanical Engineering \\ Slovak University of Technology, Nám. Slobody 17, 81231 Bratislava, Slovak Republic \\ e-mail: branislav.hucko@stuba.sk,e-mail: uhercik.filip@gmail.com
}

\begin{abstract}
The paper deals with controlling an upper limb prosthesis based on the measurement of myoelectric signals (MES) while drinking. MES signals have been measured on healthy limbs to obtain the same response for the prosthesis. To simulate the drinking motion of a healthy upper limb, the program ADAMS was used, with all degrees of freedom and a hand after trans-radial amputation with an existing hand prosthesis. Modification of the simulation has the exact same logic of control, where the muscle does not have to be strenuous all the time, but it is the impulse of the muscle which drives the motor even though the impulse disappears and passed away.
\end{abstract}

KEYWORDS: Simulation, amputation, upper limb prosthesis, ADAMS, controlling.

\section{INTRODUCTION}

The loss of a hand or limb is a very difficult situation for people to handle. The upper extremity is a coordinated, multi-articular system where the combined motion of each articulation contributes to the overall function. When the upper extremity is replaced with a prosthetic device, people may need to compensate for lost motion at the distal end due to excessive motion at other articulations.

The most modern myo-electrical prosthesis is known for great degrees of freedom as well as for greater conceptions of utilization than ever before. The huge emphasis is based on controlling, the shape, weight and comfort of the actual prosthesis as the patient uses the prosthesis as a part of its own body.

The study compared the compensatory motion of persons using a trans-radial prosthesis without wrist motion to non-amputees under unrestricted and restricted (braced) forearm rotation conditions. they performing four activities with these conditions; drinking from a cup, opening a door, lifting a box and turning a steering wheel [1].

A standardized measurement method for unconstrained movement analysis of the upper extremity, including hand orientation for a set of four daily activities and obtains normative joint angles values of the wrist, elbow, shoulder and scapula, analyzed in [2]. The joint angles of the wrist, elbow, shoulder and scapula were analyzed and measured using stereophotogrammatic recording of active LED -markers using a system with three cameras.

The investigated movements of forearm flexion/extension, forearm pronation/supination, wrist flexion/extension and wrist adduction/abduction were made in [3]. For each movement, the instantaneous axis of relative rotation had been deduced using mathematical expressions. All the geometrical and kinematic constraints allow performing the spatial movement of the upper limb in a very realistic way.

A quantitative and qualitative evaluation method for unconstrained movement analysis of the upper extremity movement followed and approximate optimal kinematic performance trajectory was analyzed in [4].

The range of motion of the upper limb of a healthy subject performing activities of daily living used for simulation was taken in [2]. 
The static and dynamic evaluation tests confirm the system accuracy and precision in describing 3D upper extremity motion [5].

Described components of an EMG driven model of the upper extremity and the experiment and data analysis required to estimate optimal muscle lengths and muscle specific tension for elbow flexors [6].

The aim of this work is to simulate daily motion of a healthy hand with all necessary degrees of freedom and to simulate the hand movement after trans-radial amputation with an existing prosthesis of the hand. Moreover, finding out how limiting the prosthesis is for the patient can be beneficial for the prosthesis control as well as its capacity for utilization. Finally, constructing a model in the ADAMS program for the simulation of healthy extremity movement.

\section{VIRTUAL MODEL OF UPPER EXTREMITY}

The human hand is a mechanism with 27 degrees of freedom. Analysis of the upper limb virtual model using ADAMS/View includes the creation of all segments, adding the bones and removing degrees of freedom from the model. Control of these movements by use of sensors and various functions are also described.

The principle of designing a virtual model of the human upper limb is to analyze some dynamics response characteristics during movement. The upper limb is divided into component segments; the segment link lengths, weights, and the location of their center of mass [7] moments of inertia [9]. The hand joint ranges are defined [8].

The human hand is a very complex tool. It is capable of performing many different grasping motions. The aim of this work is to simulate activities of daily living of upper limb movement: drinking from a cup. The definition of the power grip movements, the mechanics of gripping and the way in which the geometry of a cylindrical handle surface and phalange contacts are defined [8].

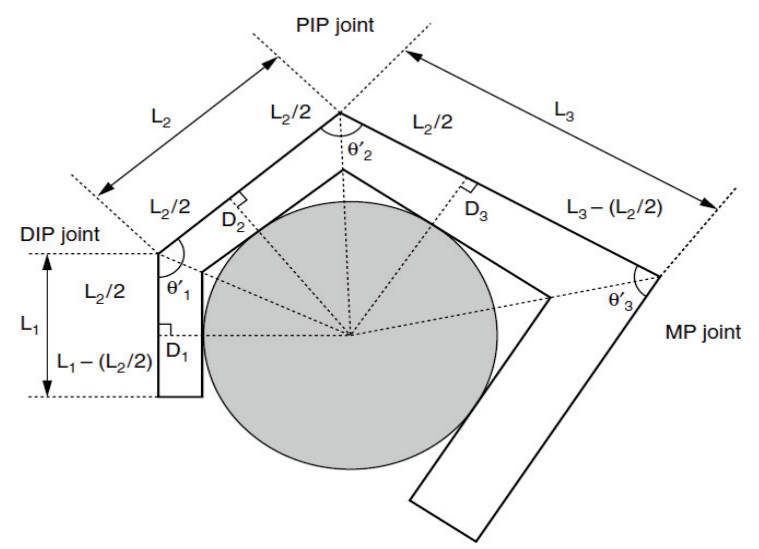

Fig. 1 Schematic diagram of power grip

\section{SIMULATION OF HEALTHY UPPER LIMB}

The power grip of taking a glass and drinking it is made in ADAMS/View software. The driver joints in this simulation are the hand joints. All the steps and angles of the joints are described in the simulations script. For this simulation two types of control were designed:

The first is the Simulation Script. We can manage simulation parameters like the velocity, acceleration, function of movements or activate and deactivate the sensors through commands. 


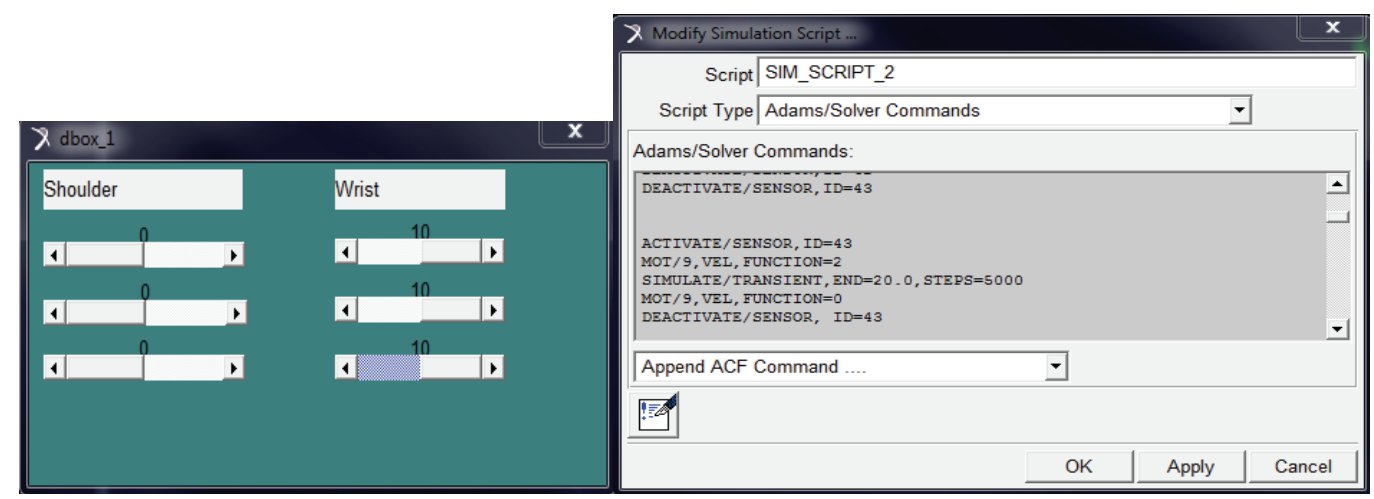

Fig. 2 The sliders and simulation script

The second is the combination of STEP functions and Sliders. This allows controlling the movements more easily than by using the simulation script. Creation of the Design Variable with a combination of Sliders allows us to keep the natural range of movements for all the segments. The contact between the hand and the glass is defined by normal force and friction. The skin coefficient of friction ranged from approximately 0.25 to 0.55 . The coefficient is inversely related to the pinch force applied by the digits, decreasing approximately 0.1 per each $30 \mathrm{~N}$ of pinch force [8].

\section{SIMULATION OF PROSTHETIC HAND MOVEMENT}

The modifications of the healthy upper limb and hand to the limb with the trans-radial (below elbow) level of amputation were made in ADAMS/View. The Michelangelo hand from Otto Bock is used for modification.

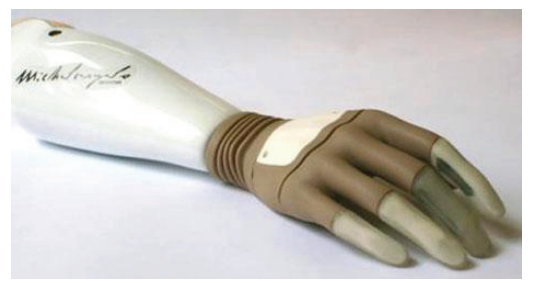

Fig. 3 Michelangelo hand

The prosthesis is capable of performing a thumb-four finger grasp and lateral grasp. The thumb only has two degrees of freedom. The fingers and wrist have only one DOF.

This simulation is therefore based on the system of control also used in the Michelangelo prosthesis. Here, an important part is focused on the potential of the muscle, meaning every contraction of the muscle has a certain potential measured by EMG electrodes. In order to control the simulation properly, it is important to control the height altogether with the intensity of muscle contraction. For a change in permanently used parts there has to be a co-contraction (a fast contraction of both muscles at the same time). The modification of simulation has exactly the same logic for control, where the muscle does not have to be constantly strained, but it is the impulse of the muscle which drives the motor even though the impulse disappeared and passes away. The motor still stays at the same position. Switching of the particular motors in the simulation is supported by simulation script which was provided. The combination of associated simulation 
script altogether with the step function provides us with the particular switching of modes, meaning particular rotators.
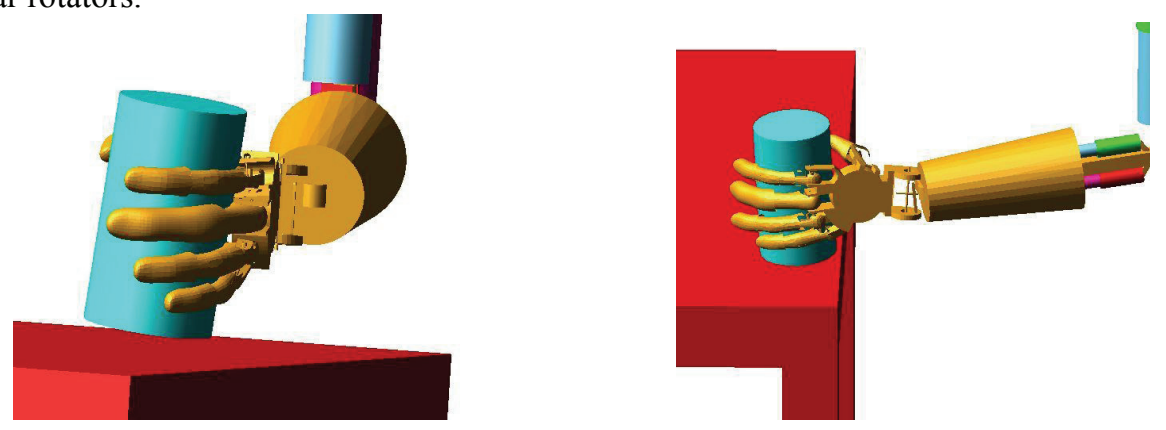

Fig. 4 The Michelangelo prosthesis

The prosthesis is controlled by the muscles instead of the revolute joint and rotational motion like it was used in the previous simulation. The rotational motion in the joint is replaced by translation in the muscle. The muscle is created by two cylinders where the first one is joined to the second one. By using the translational joint motion we can simulate the contraction of the muscle. The prosthetic hand is jointed to the muscle by a COUPLER that creates coupling of the translational and rotational motion of two or three joints. The driver joint are the translational joint in the muscle and the couple at the fingers, respectively the wrist joint. In this simulation the role of the amplifier includes the coupler function where we can regulate the ratio of the movements between the two joints with one another.

\section{SIMULATION RESULTS}

The concept of this simulation was to create the model of upper extremity using the simulation Script for controlling the movements while drinking. The angles of all the joints are defined in the Sliders and the range of movements are limited by using the Design Variables. When the movement was created the measurement of the muscular contractions could begin. The contractions of the muscles are the results of this simulation.
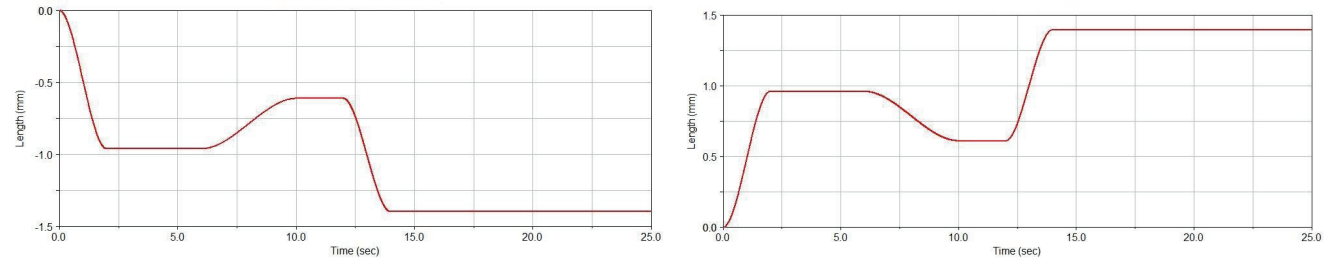

Fig. 5 The contraction of anterior and posterior forearm muscle

The graphs show the contraction of an anterior and posterior forearm muscle while drinking.
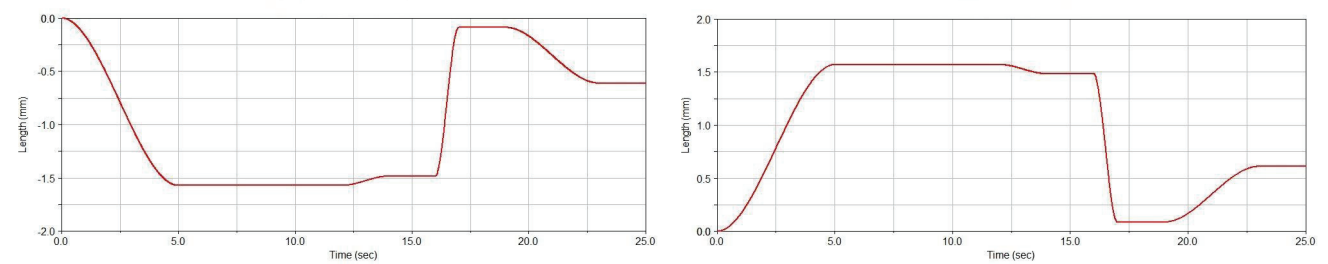

Fig. 6 The contraction of anterior and posterior forearm muscle for wrist rotating 
The contraction of muscles show how a muscle needs to be contracted to be able to get to the position when the cup is near the mouth.

The simulation is divided into 3 phases: The first one represents pronation and picking up the glass. The second phase describes the movement of the hand while rotating in order not to spill the drink, and the last phase of the movement is represented by the contraction of the muscle for drinking motion.

\section{MICHELANGELO HAND PROSTHESIS}

Simulation has also been divided into three phases that come from anatomical position. From this position it is challenging to fulfill the co-contraction in order to get the wrist rotator shifted to finger rotator for titling the cup and develop sufficient contraction of the muscle for holding the cup. Consequent co-contraction in the second phase allows for rotation of the wrist in order to offset the cup for the third phase, drinking.
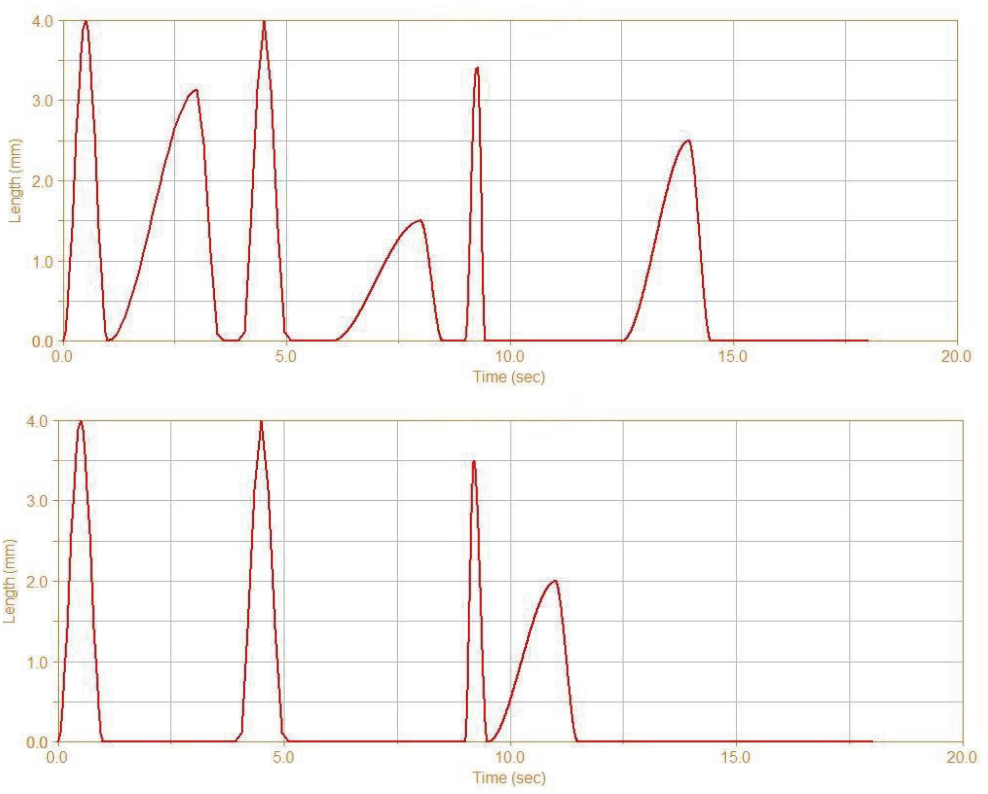

Fig. 7 The anterior and posterior muscle respectively

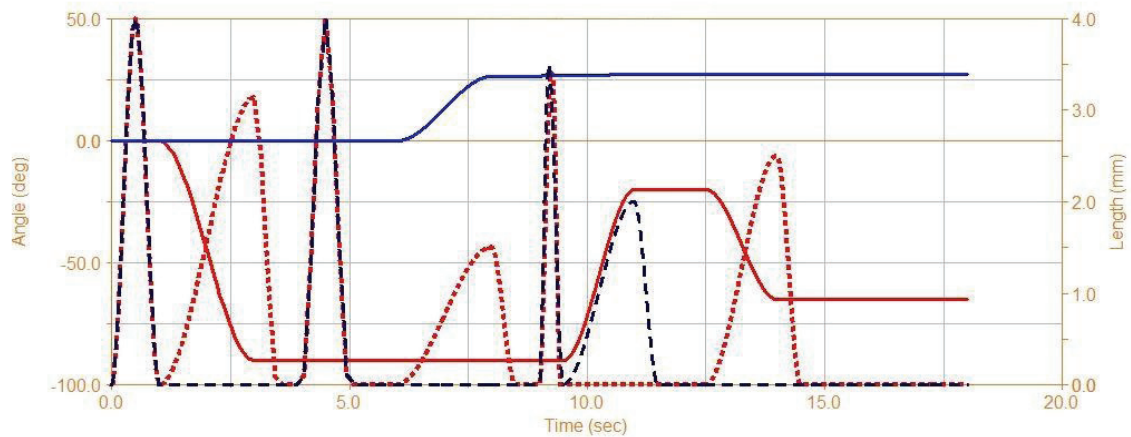

Fig. 7 The muscle contractions and wrist and finger angles 
The Fig. 8 shows precise contractions and co-contractions of the muscles and rotation angles in the wrist rotator plus fingers during the drinking phase.

The blue dashed line represents the contraction of the posterior forearm muscle. The main exercise that caused this muscle is supination, it means rotation of the wrist in the second phase, the phase where the glass is going from the table to the height of the mouth.

The red dashed line illustrates the contraction of the anterior forearm muscle. This muscle is the main creator of the most movements of the prosthesis hand. The flexion of the fingers and the pronation of the hand while drinking.

The red solid line is at first the reaction to the anterior muscle contraction in the beginning of the first phase. The wrist rotator must be removed about 90 degrees. Then, it responds to the posterior forearm contraction in the second phase of movement. In the end of the simulation it corresponds to the anterior part again.

The blue solid line is the illustration of the change in finger angles. In this simulation the fingers are moved only in the first phase where picking up the glass.

\section{CONCLUSIONS}

In this paper we are concerned with two models of the upper extremity. The first model is designed as an extremity of a healthy hand with real measurements and all of the 27 degrees of freedom. For this model we developed two varieties of control. The first variety is concerned with the simulation script, and was not very effective since rotating angles needed to be changed to rewrite part of the script. The second variety of control is concerned with the creation of the step function while using sliders which help us change angles in the simulation. The disadvantages are in the number of sliders necessary for a particular joint as the complexity of the movement cannot move backward, but the steps taken have to be the same and only then can we add or subtract values.

The constructed model with prescribed movement has been used to supplement the muscles, and by using a coupler, we could obtain the muscle contractions. As a result we have obtained the pronation and supination contractions of forearm muscles in the wrist, and flexion and extension in the finger.

Both varieties are sufficient enough to give the same results; of course for simplicity we choose the second variety. The second model was modified from a previous simulation for the amputee hand with the help of Michelangelo, Otto Bock prosthesis. Most of the joint axes, lengths, and angles of particular segments are concerned with the CATIA system and insufficient information were the reason for changing certain defined measurement.

In this model motion was generated on the basis of muscle contractions (translational movement between the two synchronized cylinders). The result of these simulation combinations are graphs of ideal muscle contractions needed in the state of drinking. However, it is very difficult for a person to reach a prefect process of controlled muscle contractions as the muscle depends on many other factors such as gender, age, and physical or mental condition of the patient. The fact that the prosthesis is being controlled by only two sensors does not provide smooth motion therefore the cocontraction for switching the rotators is needed. The problem could be removed with increased number of sensors, but the patient would be forced to learn how to control a greater amount of muscles and their impact on the prosthesis. For example, if we would use chest muscles, we could extend the choice of controlling the motion in two parts: the chest muscles would deal with the rotation of the wrist and the forearm muscles would deal with the flexion and extension of the fingers. This study is very helpful for the sensor evaluation to improve patient rehabilitation [12]. 


\section{ACKNOWLEDGEMENT}

The supports of R\&D Department, Otto Bock Gmbh and the Slovak Ministry of Education grant VEGA 1/0571/11 are gratefully acknowledged.

\section{REFERENCES}

[1] Stephanie L. Carey, M. Jason Highsmith, Murray E. Maitland, Rijav V. Dubey, Compensatory movements of transradial prosthesis users during common tasks. Clinical Biomechanics 23 (2008) 1128-1135, Elsevier Ltd., 2008.

[2] Carolien J. van Andel, Nienke Wolterbeek, Caroline A.M. Doorenbosch, DirkJan (H.E.J) Veeger, Jaap Harlaar, Complete 3D kinematics of upper extremity functional tasks, Gait \& Posture 27 (2008) 120-127, 2007.

[3] Davide Gattamelata, Eugenio Pezzuti, Pier Paolo Valentini, Accurate geometrical constrains for the computer aided modeling of the human upper limb, Computer-Aided Design 39 (2007) 540-547, 2007.

[4] Wenbin Chen, Caihua Xiong, Xiaolin Huang, Ronglei Sun, Youlun Xiong, Kinematic ananlysis and dexterity evaluation of upper extremity in activities of daily living, Gait \& Posture 32 (2010) 475-481, 2010.

[5] Brooke Hingtgen, John R. McGuire, Mei Wang, Gerald F. Harris, An upper extremity kinematic model for evaluation of hemiparetic stroke, Journal of Biomechanics 39 (2006) 681-688, 2005

[6] Joseph Langenderfer, Suzanne LaScalza, Amy Mell, James E. Carpenter, John E. Kuhn, Richard E. Hughes, An EMG-driven model of the upper extremity and estimation of long head biceps force, Computers in Biology and Medicine 35 (2005) 25-39, 2003.

[7] Hamilton, Weimar, Luttgens, Kinesiology, Specific basis of human motion, (2008)

[8] Andris Freivalds, Biomechanics of the upper limbs, CRC press, (2004).

[9] Vladimir M. Zatsiorsky, Kinetics of human motion, (2002).

[10] Gunter R. Kanitz, Otto Bock Gmbh, Analysis of control concept for prosthetics based on pattern recognition in signals acquired by single feature electrodes, (2010).

[11] Cynthia C. Norkin, D. Joyce White, Measurement of joint motion. A guide to goniometry, (2003).

[12] Churko, J.M., Mehr, A., Linassi, A., Dinh, A., Sensor evaluation for tracking upper extremity prosthesis movements in a virtual environment, Conference proceedings : Annual International Conference of the IEEE Engineering in Medicine and Biology Society. IEEE Engineering in Medicine and Biology Society. Conference Volume 2009, 2009, pp. 2392-2395 